\title{
Relation of anterior pelvic tilt during running to clinical and kinematic measures of hip extension
}

\author{
Anthony G Schache, Peter D Blanch, Anna T Murphy
}

\begin{abstract}
Background-Limited hip extension flexibility due to tight hip flexor musculature or anterior hip capsular and ligamentous structures is a possible cause of increased anterior tilt of the pelvis during running. However, to date, research exploring this relation, as well as the kinematic relation between anterior tilt of the pelvis and peak hip extension range of motion during running, is not available.

Objective-To assess the relation of anterior pelvic tilt during running to peak hip extension range of motion measured during running and hip extension flexibility measured clinically.

Methods-Hip extension flexibility was assessed using the Thomas test, and the three dimensional kinematic motion of the pelvis and hips were recorded using a VICON motion analysis system with 14 elite athletes running on a treadmill at 20 $\mathbf{k m} / \mathbf{h}$.
\end{abstract}

Results-Anterior pelvic tilt displayed a significant $(p<0.01)$ correlation with peak hip extension range of motion during running. Anterior pelvic tilt tended to be increased in runners who displayed reduced absolute peak hip extension range of motion during terminal stance. No significant correlation was shown for hip extension flexibility with either anterior pelvic tilt or peak hip extension range of motion during running.

Conclusions-The outcomes of this study indicate that anterior pelvic tilt and hip extension are coordinated movements during running. Static hip extension flexibility measured using the modified Thomas test does not appear to be reflective of these dynamic movements.

(Br F Sports Med 2000;34:279-283)

Keywords: running; kinematics; pelvis; hip; flexibility

Paediatric and Child

Health/Human

Movement Studies

Departments,

University of

Queensland, Brisbane,

Q1d, Australia

A T Murphy

Correspondence to:

Mr A G Schache, School of

Physiotherapy, University of

Melbourne, 200 Berkeley St,

Carlton, Victoria 3053,

Australia

email: a.schache@

pgrad.unimelb.edu.au

Accepted for publication

29 February 2000 Injuries to the lumbo-pelvic-hip complex account for about $14 \%$ of all injuries sustained by distance runners and sprinters of varying levels of ability. ${ }^{1}$ Although at first this injury rate appears quite low with respect to the knee and lower leg, injuries to the lumbo-pelvic-hip complex from a clinical point of view are often quite debilitating and demand prolonged periods of rehabilitation. This is evident from several case studies describing running injuries to this region. ${ }^{2-4}$ One factor that has been anecdotally linked to running related injuries of the lumbo-pelvic-hip complex is increased anterior pelvic tilt. ${ }^{56}$ It has been suggested that such a position of the pelvis is associated with an increase in the degree of lumbar lordosis during running. ${ }^{78}$ The resulting repetitive impingement of the vertebral facets from the hyperextension of the lumbar spine is then thought to be related to the onset of low back pain in runners. ${ }^{89}$

Tightness of the hip flexor musculature-for example, iliopsoas, tensor fascia lata, rectus femoris-hip joint capsule, or surrounding anterior hip ligamentous and fascial structures in runners may reduce hip extension flexibility. This is commonly assessed clinically using the Thomas test (or modifications of it). Limited hip extension flexibility has been proposed as one possible cause of increased anterior pelvic tilt and lumbar lordosis during running. ${ }^{5-7}$ Given the proposed implications of increased anterior pelvic tilt and lumbar lordosis during running, it would be of interest to explore the relation between hip extension flexibility and dynamic measures of anterior pelvic tilt and peak hip extension range of motion (ROM) during running. Published research into the relations between these three variables is not currently available. Therefore the purpose of this research project was to assess the relation of anterior pelvic tilt during running to peak hip extension ROM measured during running and hip extension flexibility measured clinically.

\section{Methods}

Fourteen (10 male, 4 female) elite track and field athletes, who were not suffering from any musculoskeletal injuries, volunteered as subjects for this study. All were experienced treadmill runners. They had a mean age of 23.6 years (range 18-29 years). Male subjects had a mean height of $177.1 \mathrm{~cm}$ (range 167.4-192.0 $\mathrm{cm}$ ), and female subjects had a mean height of $167.8 \mathrm{~cm}$ (range $163.6-174.5 \mathrm{~cm}$ ). The study was conducted in accordance with the guidelines of the Australian Institute of Sport ethics committee.

Hip extension flexibility was measured using

the Thomas test. For this test, each subject sat on the end of a plinth and rolled backwards on to the plinth while holding both knees to the chest. This position ensured that the pelvis was posteriorly tilted and the lumbar spine flexed on the plinth. Tester one held one hip in maximal flexion to maintain this pelvic position, while the limb to be measured was lowered towards the floor with the knee in a relaxed position. The head and shoulders of each subject remained in a flat position on the plinth throughout the test. Tester two used a goniometer with a spirit level attached to the arm to 


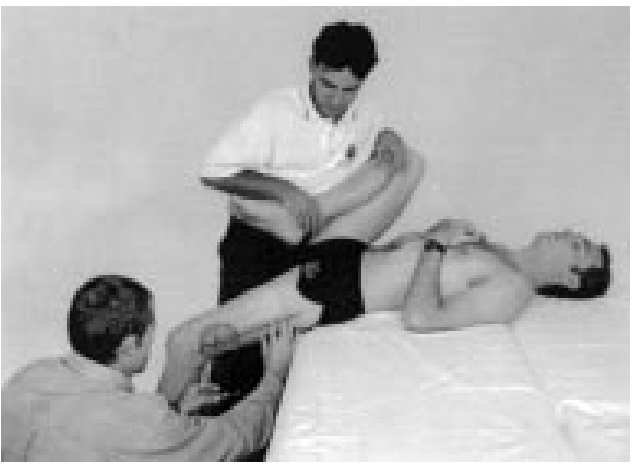

Figure 1 Measurement of hip extension flexibility using the Thomas test.

measure the angle formed between a horizontal reference line and a line connecting the greater trochanter to the lateral femoral epicondyle (fig 1). A positive value was assigned to the situation where the thigh was extended below the horizontal reference line. This test has been previously found to be adequately reliable when measuring normal subjects. ${ }^{10}$

All running sessions were performed on a treadmill (Sportech Gymnasium and Electronic Sports Equipment, Jamison, ACT, Australia) custom made for the Australian Institute of Sport biomechanics laboratory. The treadmill was set with no incline. To measure the motion of the pelvis and hips during running, small reflective markers were positioned on both anterior superior iliac spines, the midpoint between the two posterior superior iliac spines and both lateral femoral condyles. In addition, two markers mounted on small wands were positioned on the lateral aspect of the distal third of the right and left thighs on a line connecting the lateral projection of the hip joint centre (greater trochanter) to the lateral projection of the knee joint centre (lateral femoral condyle). All markers were positioned according to the VICON Clinical Manager (Oxford Metrics Ltd, Oxford, UK) protocol.

Each subject performed a five minute warm up running on the treadmill at the test speed of $20 \mathrm{~km} / \mathrm{h}$. After this period, the three dimensional trajectories of the markers were collected using a VICON motion analysis system (Oxford Metrics Ltd) with six cameras (NAC Inc, Yokohama, Japan) operating at a sampling rate of $200 \mathrm{~Hz}$. Foot strike and foot off were detected using a load cell (Applied Measurement Australia Pty Ltd, Jamison, ACT, Australia) attached to the treadmill.

Immediately after the five minute warm up, five seconds of data were captured for each subject, and a single representative cycle was chosen for analysis. The three dimensional angular rotations of the pelvis and hips were computed using a technique equivalent to the geometrical conventions described by Grood and Suntay. ${ }^{11}$ Pelvic motion was measured as a rotation of the pelvic segment with respect to a global coordinate system (laboratory). Hip motion was measured as a rotation of the thigh segment with respect to the pelvic segment (fig 2). More specifically, pelvic tilt was defined as a rotation of the pelvic segment about the medial-lateral axis of the global coordinate sys-

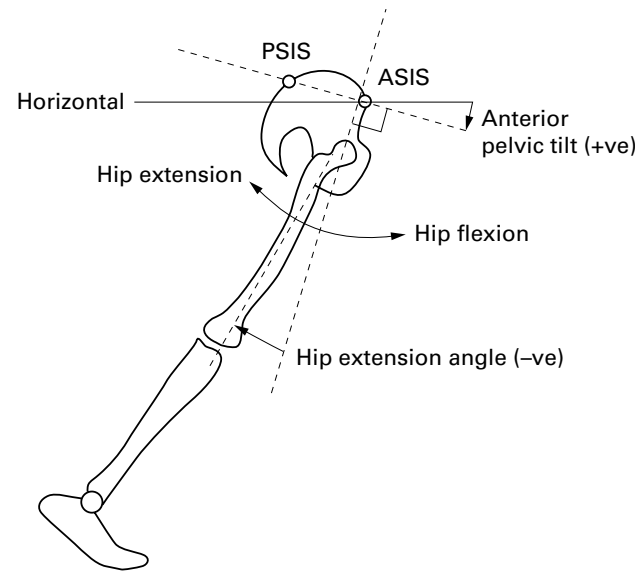

Figure 2 Diagrammatic representation of the measurement of anterior pelvic tilt and peak hip extension range of motion during running. PSIS, posterior superior iliac spine; ASIS, anterior superior iliac spine.

Table 1 Group means and ranges for measured variables

\begin{tabular}{lll}
\hline Variable & Mean & Range \\
\hline Anterior pelvic tilt $\left(^{\circ}\right)$ & 22.1 & $13.6-37.0$ \\
Peak hip extension ROM $\left(^{\circ}\right)$ & -11.7 & $-27.1-7.5$ \\
Hip extension flexibility $\left(^{\circ}\right)$ & 17.4 & $7.5-25.0$ \\
Relative stride length $(\%)$ & 206.3 & $186.8-216.4$ \\
Relative leg length $(\%)$ & 53.5 & $51.1-56.1$ \\
\hline
\end{tabular}

ROM, range of motion.

tem (rotation occurring in the sagittal plane of the global coordinate system). Hip flexion and extension was defined as a rotation of the thigh segment about the medial-lateral axis of the local coordinate system in the pelvic segment (rotation in the sagittal plane of the pelvic coordinate system). Anterior pelvic tilt was assigned a positive value, and true hip joint extension was assigned a negative value in this study.

\section{DATA ANALYSIS}

Anterior pelvic tilt was measured as the angle of the pelvis at the time of terminal hip extension. The magnitude of terminal hip extension was used to represent peak hip extension ROM. Stride time, based on the information from the load cell, represented the time between two foot strikes on the same side of the body. Stride length was calculated by multiplying the known stride time by the belt speed of the treadmill. Relative stride length and relative leg length for each subject were then obtained by expressing the values as a percentage of the respective height.

Data for the left and right sides were compared using a two tailed paired $t$ test. For each subject, data for the left and right sides were combined, and average values were used in analyses. Means and ranges were calculated for each of the variables. Simple and multiple regression analyses were used to assess the relations between all of the variables.

\section{Results}

Individual $t$ tests showed no significant differences between the left and right sides for any of the variables $(p>0.05)$. Therefore the use of average values of the left and right side for each subject was considered appropriate. Table 1 
Table 2 Regression analysis results

\begin{tabular}{llllll}
\hline & $\begin{array}{l}\text { Anterior } \\
\text { pelvic tilt } \\
(\text { APT })\end{array}$ & $\begin{array}{l}\text { Peak hip } \\
\text { extension ROM } \\
\text { (HEROM) }\end{array}$ & $\begin{array}{l}\text { Hip extension } \\
\text { flexibility (HEF) }\end{array}$ & $\begin{array}{l}\text { Relative stride } \\
\text { length (RSL) }\end{array}$ & $\begin{array}{l}\text { Relative leg } \\
\text { length (RLL) }\end{array}$ \\
\hline APT & - & ${ }^{\star \star} \mathrm{y}=0.6 \mathrm{x}+30$ & $\mathrm{y}=-0.05 \mathrm{x}+23$ & $\mathrm{y}=-0.2 \mathrm{x}+68$ & $\mathrm{y}=1.9 \mathrm{x}-77$ \\
HEROM & $\mathbf{0 . 8}{ }^{\star \star}$ & - & $\mathrm{y}=-0.1 \mathrm{x}-10$ & $\mathrm{y}=-0.4 \mathrm{x}+63$ & ${ }^{\star} \mathrm{y}=3.7 \mathrm{x}-209$ \\
HEF & $\mathbf{0 . 0 0 2}$ & $\mathbf{0 . 0 0 4}$ & - & $\mathrm{y}=0.07 \mathrm{x}+3$ & $\mathrm{y}=0.1 \mathrm{x}+10$ \\
RSL & $\mathbf{0 . 0 8}$ & $\mathbf{0 . 1 2}$ & $\mathbf{0 . 0 1}$ & - & $\mathrm{y}=-1 \mathrm{x}+271$ \\
RLL & $\mathbf{0 . 1 5}$ & $\mathbf{0 . 3 1 ^ { \star }}$ & $\mathbf{0 . 0 0 1}$ & $\mathbf{0 . 0 4}$ & - \\
\hline
\end{tabular}

Shaded numbers are the $\mathrm{R}^{2}$ values and the rest are the regression equations.

${ }^{\star}$ Significant result at $\mathrm{p}<0.05 ;{ }^{\star \star}$ significant result at $\mathrm{p}<0.01$.

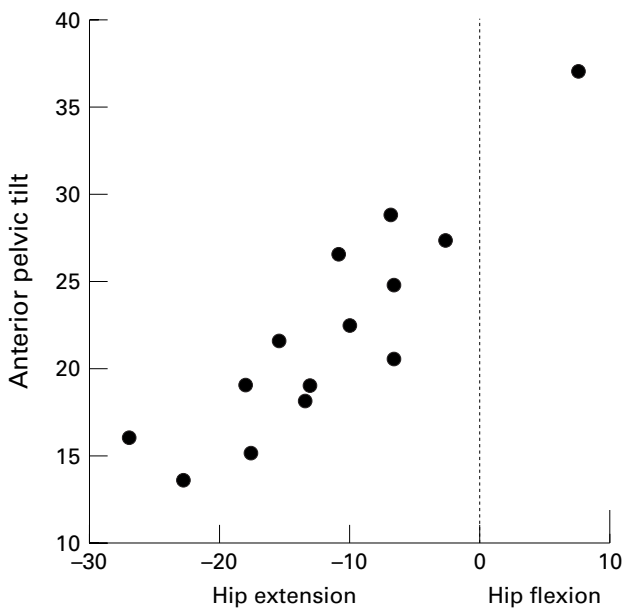

Peak hip extension ROM

Figure 3 Anterior pelvic tilt versus peak hip extension range of motion (ROM) in the 14 subjects. Both variables are reported in degrees.

shows the group means and ranges for each measurement, and table 2 the results from the simple regression analysis.

Anterior pelvic tilt was found to have a significant $(\mathrm{p}<0.01)$ positive correlation with peak hip extension ROM during running. This meant that anterior pelvic tilt tended to be increased in runners who displayed reduced absolute peak hip extension ROM during terminal stance (fig 3). Peak hip extension ROM was found to have a weak but statistically significant $(p<0.05)$ positive correlation with relative leg length. Subjects with increased relative leg lengths tended to run with reduced absolute peak hip extension ROM. No significant correlation was found between anterior pelvic tilt and hip extension flexibility. Also, no significant correlation was found between peak hip extension ROM during running and hip extension flexibility.

A stepwise multiple regression was performed as an additional analysis in which anterior pelvic tilt was the dependent variable and peak hip extension ROM, hip extension flexibility, relative stride length, and relative leg length were the independent variables. The equation with maximal prediction accuracy using the least amount of independent variables contained only peak hip extension ROM, as hip extension flexibility, relative stride length, and relative leg length were all found to make insignificant contributions.

\section{Discussion}

The average values of $22.1^{\circ}$ and $-11.7^{\circ}$ for anterior pelvic tilt and peak hip extension
ROM respectively compare favourably with three dimensional graphical data of running reported by Novacheck. ${ }^{12}$ The average hip extension flexibility value of $17.4^{\circ}$ is also similar to the results of Harvey, ${ }^{13}$ who found a mean value of $14^{\circ}$ for a group of runners.

Anterior pelvic tilt was found to be related to peak hip extension ROM during running (fig 3 ). Figure 3 shows the increasing pelvic tilt angle in subjects with reduced absolute peak hip extension ROM. Figures 4 and 5 show the sagittal plane movements of the pelvis and hips over a running cycle for the subject displaying the greatest degree of absolute peak hip extension ROM (fig 4) and the subject displaying the smallest degree of absolute peak hip extension ROM (fig 5). On comparison of figs 4 and 5, the anterior pelvic tilt angle can be seen to differ by about $20^{\circ}$ between the two subjects. A significant positive correlation between anterior pelvic tilt and peak hip extension ROM has also been found by Lee and coworkers, ${ }^{14}$ who investigated the walking pattern of 41 neurological patients with bilateral hip flexion contractures.

When viewing figs $3-5$ it is apparent that the sagittal plane movement patterns of the pelvis and hips differ across a group of subjects running at the same speed. Wilson and coworkers, ${ }^{15} 16$ when investigating the kinematic behaviour of the knee, showed that the joint followed a unique path of least resistance that was determined by the geometrical configurations of the joint surface and surrounding anatomical structures. This led Nigg and coworkers ${ }^{17}$ to propose that the skeleton has a preferred path for a given movement task-for example, running. Based on this, the differing sagittal plane movement patterns of the pelvis and hips displayed in this study may be explained on the basis of individual variations in the preferred paths of motion of the involved joints. Some subjects displayed a preferred path during terminal stance that involved predominantly hip extension, whereas others displayed a preferred path that involved increased anterior pelvic tilt with less hip extension. As the subjects were all running at the same velocity, the differences between the various paths in terms of forward momentum may be minimal. For example, the thigh segment measured with respect to the global coordinate system may well be similar for different paths. The question that is of vital importance is whether a particular path is related to injury. The anecdotal literature to date suggests that increased anterior pelvic tilt during running is related to injury. ${ }^{56}$ Further research is required to support or negate these hypotheses.

It has been reported that anatomical features may actually provide the limit to hip extension during running, as maximal values appear to approach the limit for passive range. ${ }^{18}$ From a clinical point of view, it would seem logical that the flexibility of the soft tissue structures anterior to the hip is a factor that determines the preferred path of motion - that is, a relation between peak hip extension ROM and hip extension flexibility exists. However, hip exten- 


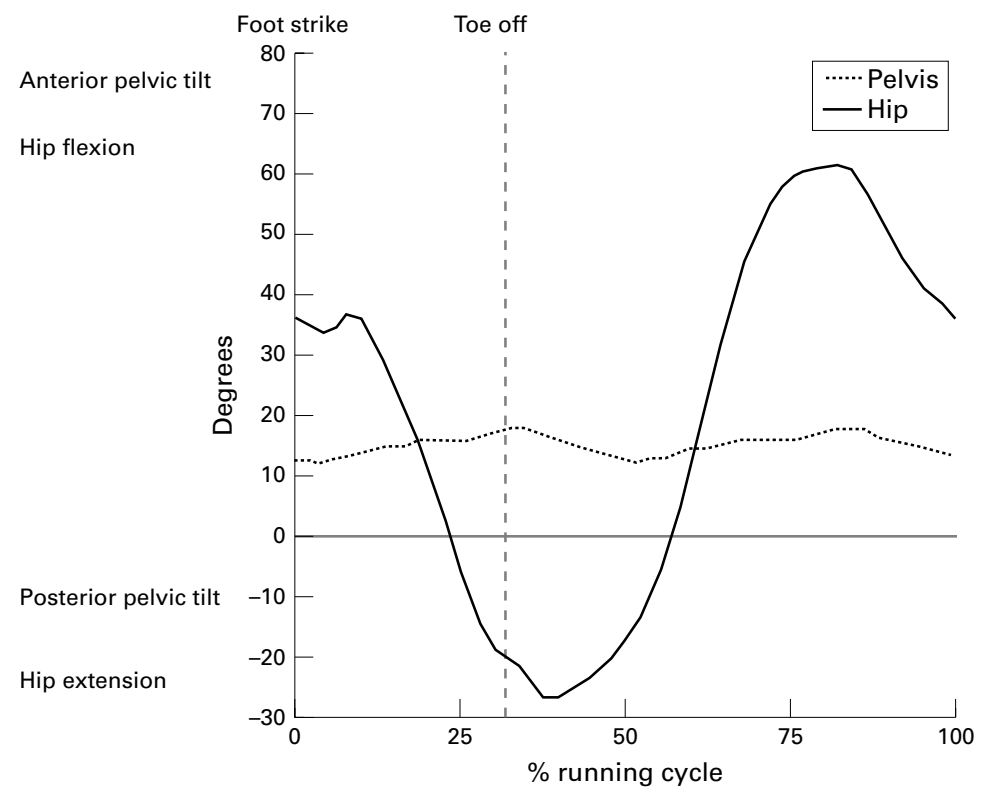

Figure 4 Sagittal plane pelvis and hip angles over the running cycle for the subject displaying the greatest degree of peak hip extension range of motion.

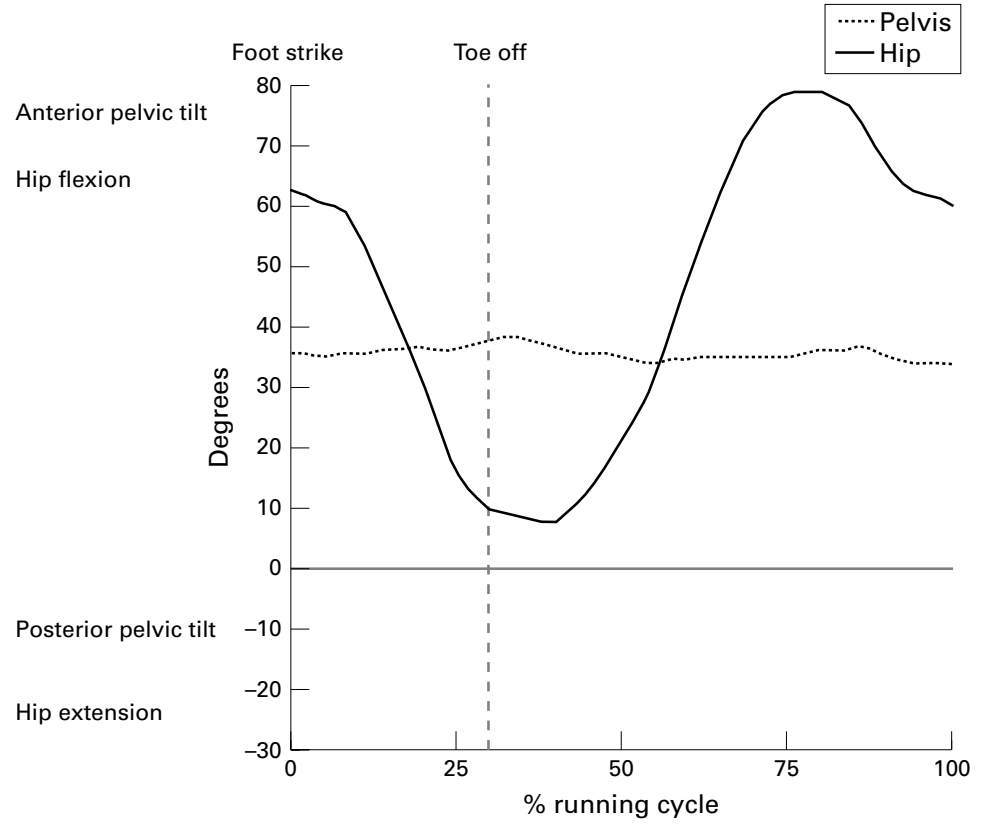

Figure 5 Sagittal plane pelvis and hip angles over the running cycle for the subject displaying the smallest degree of peak hip extension range of motion.

sion flexibility, measured using the Thomas test described in this study, was not found to be indicative of the dynamic measures of peak hip extension ROM or anterior pelvic tilt.

The results of this study compare favourably with the work of others. Several researchers ${ }^{14} 1920$ have reported similar findings in studies investigating the kinematic pattern of the pelvis and hips during walking in patients with varying degrees of hip flexion contractures. In addition, Bar-On and coworkers ${ }^{21}$ measured the degree of hip flexion contracture using the Thomas test on 51 subjects with neurological deficits and found no correlation with radiologically determined measures on the same subjects. As a result of the findings from this study and the work of others, it is advised that clinicians need to be extremely cautious about making predictions about the dynamic sagittal plane movements of the pelvis and hips based on the outcomes of the Thomas test.

There are several possible explanations as to why the Thomas test measure was not found to relate to the dynamic measures. It may be that static flexibility is not the major factor governing the degree of anterior pelvic tilt or peak hip extension ROM when running at a submaximal speed. Such variables may be determined by complex dynamic neuromotor patterns rather than static flexibility alone. It is feasible that static soft tissue restraints only become a factor at maximal speeds of running. Further research is required to answer this question. There are also differences with regard to the position of the pelvis and the external forces acting about the hip joints between the two situations. Future researchers might consider modifying the Thomas test to replicate the position of the pelvis during running.

Other clinical tests that relate to anterior pelvic tilt and peak hip extension ROM during running need to be investigated. For example, Ounpuu and coworkers ${ }^{19}$ measured 44 patients with cerebral palsy, and found a high positive correlation between pelvic position in the sagittal plane during standing and dynamic measures of pelvic tilt during walking. Clinical measures of the pelvic tilt angle in standing are easy to perform and have certainly been shown to be reliable and valid. ${ }^{22-24}$ If it could be shown that relations between pelvic tilt in standing and dynamic measures of anterior pelvic tilt and peak hip extension ROM during running exist, then the practitioner would have a simple test that is reflective of the dynamic sagittal plane movements of the pelvis and hips. The ultimate clinical relevance of this test though depends on whether a particular dynamic sagittal plane movement pattern of the pelvis and hips is shown to be related to injury.

Peak hip extension ROM was found to have a positive correlation with relative leg length. This meant that subjects with increased relative leg lengths tended to run with reduced peak hip extension ROM. This may well be a product of having different sized people running at the same absolute velocity on a treadmill. Subjects with longer relative leg lengths had longer levers to run with and therefore required less peak hip extension ROM than those with smaller relative leg lengths. One may also expect subjects in this study with longer relative leg lengths to run with smaller relative strides; however, a correlation between relative leg length and relative stride length was not found.

The relatively small sample size must be taken into account when reviewing the results of this study. Also, the findings relate specifically to treadmill running at $20 \mathrm{~km} / \mathrm{h}$ and the technique used to assess hip extension flexibility. Future research is required to establish whether similar results would be obtained in a larger population running overground at different speeds.

In conclusion, anterior pelvic tilt and hip extension appear to be coordinated movements 
during the running action. Static hip extension flexibility, measured using the Thomas test, was not found to be reflective of these dynamic movements. The ability for clinicians to interpret the results of the Thomas test with regard to the sagittal plane movement patterns of the pelvis and hips during running may therefore be limited.

This project was funded by a grant from the Australian Olympic Athlete Program. The research was performed while A G S and A T M were working in the physiotherapy and biomechanics departments respectively at the Australian Institute of Sport.

1 Bennell KL, Crossley K. Musculoskeletal injuries in track and field: incidence, distribution and risk factors. Aust $\mathcal{F ~ S c i}$ Med Sport 1996;28:69-75.

2 Koch RA, Jackson DW. Pubic symphysitis in runners: a report of two cases. Am 7 Sports Med 1981;9:62-3.

3 Fields KB, Kramer JS, Delaney MJ. Osteitis pubis and pelvic stress fracture in an elite female distance runner. Clin Sports Med 1990;2:173-8.

4 Rold JF, Rold BA. Pubic stress symphysitis in a female distance runner. Physician and Sports Medicine 1986;14:61-5.

5 Geraci MCJr. Overuse injuries of the hip and pelvis. Fournal of Back and Musculoskeletal Rehabilitation 1996;6:5-19. 6 Klein KK, Roberts CA. Mechanical problems of marathoners and joggers: cause and solutio

7 Bach DK, Green DS, Jensen GM, et al. A comparison of muscular tightness in runners and nonrunners and the relation of muscular tightness to low back pain in runners. F Orthop Sports Phys Ther 1985;6:315-23.

8 Slocum DB, James SL. Biomechanics of running. FAMA 1968;205:721-8.

9 Jackson DW, Sutker AN. Low back problems in runners. In: Mack RP, ed. American Academy of Orthopaedic Surgeons symposium on the foot and leg in running sports. St Louis: CV Mosby Co, 1982:123-34.

10 Bartlett MD, Wolf LS, Shurtleff DB, et al. Hip flexion contractures: a comparison of measurement methods. Arch Phys Med Rehabil 1985;66:620-5.
11 Grood ES, Suntay WJ. A joint coordinate system for the clinical description of three-dimensional motions: application to the knee. F Biomech Eng 1983;105:136-44.

12 Novacheck TF. The biomechanics of running. Gait and Posture 1998;7:77-95.

13 Harvey D. Assesment of the flexibility of elite athletes using the modified Thomas test. Br F Sports Med 1998;32:68-70.

14 Lee LW, Kerrigan DC, Della Croce U. Dynamic implications of hip flexion contractures. Am $\mathcal{F}$ Phys Med Rehabil 1997;76:502-8.

15 Wilson DR, Feikes AB, Zavatsky AB, et al. The one degreeof-freedom nature of the human knee joint. Basis for a kinematic model. In: Hoffer JA, Chapman A, Eng JJ, et al, eds. Proceedings of the Ninth Biennial Conference of the Canadian Society for Biomechanics. Vancouver: Conference Organizing Committee, 1996:194-5.

16 Wilson DR, Feikes JD, O'Connor JJ. Ligaments and articular contact guide passive knee flexion. F Biomech 1998;31: 1127-36.

17 Nigg BM, Nurse MA, Stefanyshyn DJ. Shoe inserts and orthotics for sport and physical activities. Med Sci Sports Exerc 1999;31:S421-8.

18 Williams KR. Biomechanics of running. Exerc Sport Sci Rev 1985;13:389-441.

19 Ounpuu S, Davis RB, Walsh JHP, et al. Sagittal plane pelvic motion: relationship to standing pelvic position and clinical measures. Dev Med Child Neurol 1995;37:25.

20 DeLuca PA, Ounpuu S, Davis RB, et al. Effect of hamstring and psoas lengthening on pelvic tilt in patients with spastic diplegic cerebral palsy. F Pediatr Orthop 1998;18:712-18.

21 Bar-On E, Malkin C, Eilert RE, et al. Hip flexion contracture in cerebral palsy. The association between clinical and radiological measurement methods. Clin Orthop 1992;281:97-100.

22 Alviso DJ, Dong GT, Lentell GL. Intertester reliability for measuring pelvic tilt in standing. Phys Ther 1988;68:1347-

23 Cromwell RD, Cummings GS, Walker JR, et al. Intratester and intertester reliability and validity of measures of innominate bone inclination. F Orthop Sports Phys Ther 1994;20:88-97.

24 Gajdosik R, Simpson R, Smith R, et al. Pelvic tilt. Intratester reliability of measuring the standing position and range of motion. Phys Ther 1985;65:169-74.

\section{Take home message}

Anterior pelvic tilt and hip extension are coordinated movements during running. Static hip extension flexibility does not appear to be reflective of these dynamic movements. Clinicians must therefore be cautious about making predictions about the sagittal plane movements of the pelvis and hips during running based on the results of the Thomas test. 